\title{
Anisotropic Fluorescence Emission and Photobleaching at the Surface of One Dimensional Photonic Crystals sustaining Bloch Surface Waves. \\ I. Theory
}

Francesco Michelotti ${ }^{1,2}$, Elisabetta Sepe ${ }^{1}$

${ }^{I}$ Department of Basic and Applied Science for Engineering, SAPIENZA University of Rome, Via Antonio Scarpa 16, 00161 Rome, Italy ${ }^{2}$ francesco.michelotti@uniromal.it

\begin{abstract}
Photonic crystal (PC) enhanced fluorescence has been proposed as a novel tool for early disease detection in a liquid biopsy format. However, photobleaching of the emitters has never been deeply investigated, despite its cross section is expected to increase due to the large field intensity enhancement. Herein, we report on a comprehensive theoretical description of the stationary fluorescence emission of molecular emitters bound at the surface of a one dimensional photonic crystal (1DPC) biosensor. The model considers coupling of the emission to the large local density of the states provided by the 1DPC, in particular to the Bloch surface waves, which can be characterized by different polarization states. The rotational diffusion equation in the presence of photobleaching was solved analytically by a Laplace spherical harmonics analytical approach. The results show that photobleaching can severely affect the fluorescence emission in terms of total intensity and polarization composition, suggesting that a careful analysis of fluorescence anisotropy in biosensing experiments with PC should be carried out. We applied the model to some case of study conditions that take place in the experiments and propose a procedure to rule out the contribution of photobleaching. Last but not least, we propose fluorescence recovery after orientational photobleaching as a new tool to study rotational diffusion of emitters, or labelled proteins, bound at a surface.
\end{abstract}




\section{INTRODUCTION}

Photobleaching (PB) is a limiting factor for the application of molecular organic emitters in biophotonics. In luminescence microscopy, the local probe concentration may change also due to PB, complicating quantitation intensity measurements. Such occurrence justifies the efforts towards luminescent dyes and sensing methods that allow excluding or compensating PB [1]. Methods that do not depend on concentration are desirable, such as ratiometric probes or lifetime-based sensing [2]. The same occurs in luminescence biosensors, which rely on the direct proportionality between the emission intensity and the concentration of antigenes captured at their surface [1]. In biosensing applications, fluorescence emitters are generally used and we shall restrict our considerations to fluorescence only, without losing generality.

While in fluorescence microscopy PB can be acceptable when investigating morphology, it cannot be neglected in biosensing, especially when the target concentration is very close to the limit of detection. It is therefore paramount to characterize PB in fluorescence biosensors in view of its management for a reliable detection [1].

Among fluorescence biosensors, plasmonic [3] and photonic crystal (PC) [4] enhanced fluorescence biosensing schemes emerged. PC were deeply investigated, due to the extreme values of their local density of the optical states (LDOS), which modifies the fluorescence excitation rates and angular emission patterns [5]. However, despite the large intensity enhancement factors provided by PC, in most of works PB was either not cited or only offhandedly discussed, except some isolated cases in which PB during the operation of PC biosensors was investigated experimentally [6], without however addressing the anisotropy effects [2] arising when the fluorescence excitation and emission are coupled to differently polarized modes of the same PC structure.

Recently, one dimensional PC (1DPC) fluorescence biosensors sustaining Bloch surface waves (BSW) were proposed as a valid alternative to surface plasmon polariton (SPP) fluorescence biosensors [7,8]. However, also in such cases, PB was never properly discussed.

Since BSW, contrarily to SPP, can be either TE or TM polarized [9] a significant portion of the emission of a fluorescent molecule can be coupled to the available BSW, either TE or TM [10]. Detecting the relative intensities of the TE and TM BSW components can therefore permit to trace back to the emitters/proteins' orientation and eventually to their kinetics under non equilibrium conditions. It is therefore of particular interest to study polarization resolved BSW coupled fluorescence emission at the surface of a 1DPC. 
Besides that, in both PC and 1DPC fluorescence biosensors, the orientational distribution of emitters is usually assumed to be isotropic, also if the excitation is polarized [11-13]. Such an assumption derives from the consideration that fluorescence depolarization takes place in a very short time scale for freely rotating emitters and compensates for deviations from the isotropic distribution caused by angularly selective PB [2,14]. Under such an assumption, PB gives only rise to an overall decrease of the intensity and the response of a biosensor can be calibrated by evaluating the PB rate [6]. However, for emitters bound at a surface, as for most of PC fluorescence biosensors, such an assumption can be wrong since rotational motion might be hindered. Evidence of such condition was found in our recent experiments, showing that PB can modify both the number of emitters and their orientational distribution [15].

Here we describe in a comprehensive theoretical frame the stationary fluorescence emission of molecular emitters bound at the surface of a 1DPC sustaining both TE and TM BSW. The theory accounts for PB and rotational diffusion of the emitters. The results should be compared to our experiments, which will be reported in a second twin article [15].

\section{MODEL 1D PHOTONIC CRYSTAL}

All results reported here were obtained for a model 1DPC with the design shown in Figure 1a. The 1DPC sustains BSW in the visible range when operating in an aqueous external medium, which is typical in biosensing. The geometry and the choice of materials result from an optimization aimed at developing highly resolved BSW label-free and fluorescence biosensors [16]. The design was used to fabricate the 1DPC used in experiments on anisotropic fluorescence emission and PB [15]. The specific 1DPC design does not affect the general validity of the present theoretical results, which could be recalculated for different cases, if needed.

The model 1DPC is constituted by a $\mathrm{SiO}_{2} / \mathrm{Ta}_{2} \mathrm{O}_{5}$ periodic part (5 layers) and a $\mathrm{TiO}_{2} / \mathrm{SiO}_{2}$ topping bilayer. The nominal thicknesses of the periodic layers are $\mathrm{d}\left(\mathrm{SiO}_{2}\right)=275 \mathrm{~nm}$ and $\mathrm{d}\left(\mathrm{Ta}_{2} \mathrm{O}_{5}\right)=120 \mathrm{~nm}$. The nominal thicknesses the bi-layer are $\mathrm{d}\left(\mathrm{TiO}_{2}\right)=20 \mathrm{~nm}$ and $\mathrm{d}\left(\mathrm{SiO}_{2}\right)=20 \mathrm{~nm}$. The substrate material is a cyclic olefin copolymer (TOPAS5013LS [17]). In the calculations we used the dispersions of the complex refractive indices measured by means of reflectance/transmittance of test samples [18].

\section{BSW ENHANCED FLUORESCENCE EXCITATION AND COLLECTION}

As sketched in Figure 1a, an excited molecule at a distance d from the 1DPC surface can fluoresce by coupling to the available BSW [19]. The coupling strength is governed by the LDOS, which can be relatively very large for BSW due to their strong localization, and in general varies with position, 
orientation, and direction of the emitter [20]. Typically, for an ensemble of isotropically oriented emitters at $\mathrm{d}=0$, a 10-20\% fraction of the fluorescence power couples to BSW and the rest is radiated into either the substrate or the cladding [10]. Here, the 1DPC was purposely designed to sustain two BSW in the visible range, with either TE or TM polarization and an excited molecule can couple to both of them.

For the moment, we assume monochromatic emitters at $\lambda_{\mathrm{MAX}}$. If they radiate into a BSW, the latter propagates along the surface and, due to the finiteness of the 1DPC, leaks into the substrate at an angle $\alpha$. Figure 1a shows the normalized transverse square field distributions of the TE- $\left(|\mathrm{E}|^{2}\right)$ and TM-BSW $\left(|\mathrm{H}|^{2}\right)$ at $\lambda_{\mathrm{MAX}}$. The fields' intensity decreases exponentially in the external medium with decay constants $h_{E M}^{T E}=$ $117 \mathrm{~nm}$ and $h_{E M}^{T M}=182 \mathrm{~nm}$, respectively. Figure 1a also shows that the TM field localization is weaker and leakage is stronger.
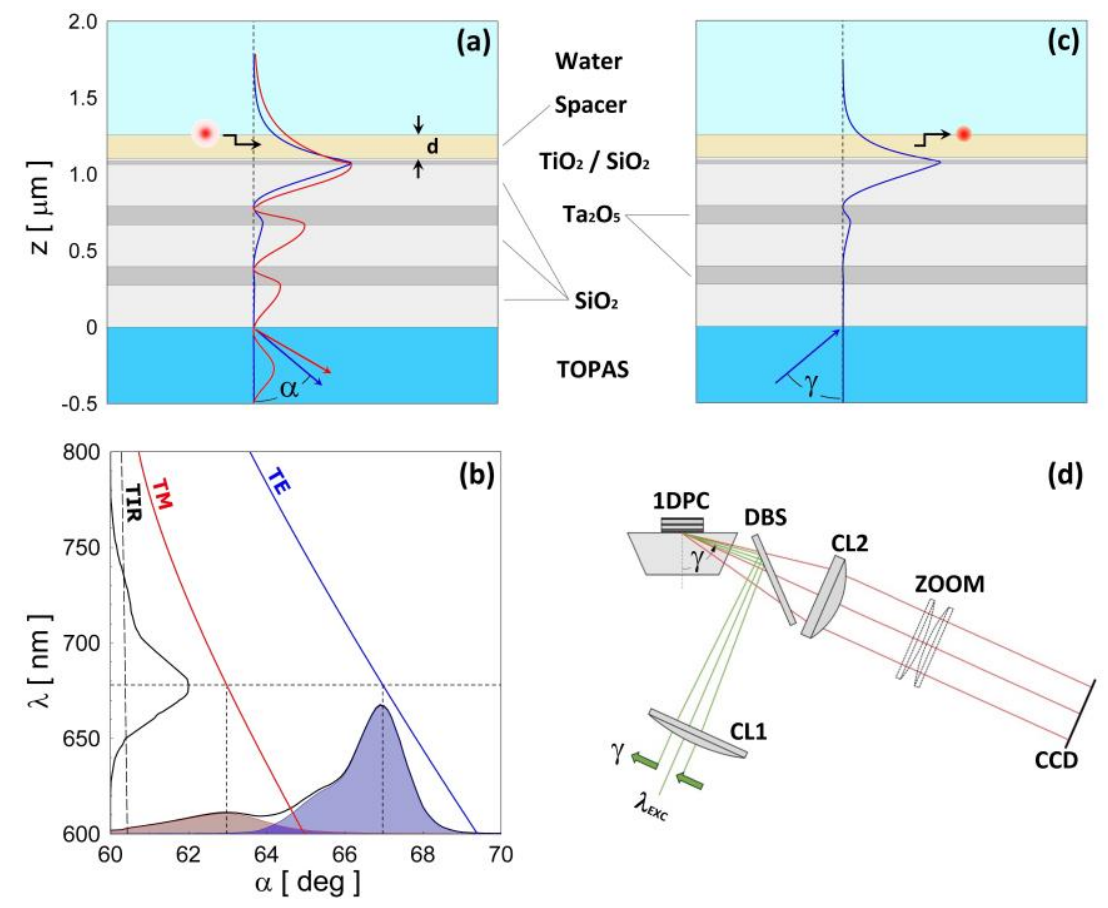

Figure 1 (color online) (a) 1DPC and emitter/BSW coupling. Transverse square field for the TE- (blue) and TM-BSW (red). (b) Calculated relationships between $\lambda$ and $\alpha$ for the TE- (blue), TM-BSW (red) and TIR (long dashed). Dylight650 free space emission spectrum (black) and resulting angular emission patterns for the TE (blue), TM (red) cases and their sum (black). (c) Coupling between an excitation field at $\gamma$ and a TE-BSW and between the TE-BSW and an emitter in the ground state. (d) Apparatus for the excitation and collection of BSW fluorescence.

Figure $1 \mathrm{~b}$ shows the dispersions for the TE-BSW (blue) and TM-BSW (red) and that the two monochromatic BSW at $\lambda_{\operatorname{MAX}}$ leak in the substrate at two different $\alpha$. In Figure 1b, we also plot the total 
internal reflection (TIR) dispersion for the TOPAS/water interface, showing that the BSW can be excited or can leak only from the substrate side in the Kretschman-Raether scheme [21]. Alternatively, the BSW dispersions can be plotted in the $(\omega, \beta)$ plane, where $\omega$ is the angular frequency and $\beta$ is the parallel component of the wave-vector. All curves reported in Figure 1 were calculated by means of a transfermatrix method [10].

If the emitters' spectrum is broad, each spectral component at $\lambda$ can couple to a BSW with different $\beta$ and $\alpha$ as shown in Figure 1b, where we plot along the ordinates the fluorescence spectrum of a specific dye molecule, Dylight650 (Thermo Fisher Scientific), and on the abscissa the resulting angular distribution of the radiation leaking into the substrate, for the TE and TM polarization and their sum. Such patterns were calculated for an isotropic angular distribution of the emitters, according to the method described more in detail below. We also assumed that the emitters are uniformly distributed in a $1 \mathrm{~nm}$ thick water layer at $\mathrm{d}=0$. The BSW dispersions play a spectroscopic role and angularly resolve the emission in terms of wavelength and polarization. It is not difficult to realize that such a feature provides a unique tool to investigate the orientational distribution of the emitters. A change of the orientational distribution will in fact modify coupling to the TE- and TM-BSW and the amplitude of the two bands.

Here we make use of the emission spectrum of the dye Dylight650, with $\lambda_{\mathrm{MAX}}=667 \mathrm{~nm}$, which was used in our experiments [15].

As far as it concerns the excitation of the emitters at the 1DPC surface, here we assume that it is carried out by means of the optical configuration shown in Figure 1c. A TE polarized laser beam at $\lambda_{\mathrm{EXC}}=637 \mathrm{~nm}$, illuminates the 1DPC from the substrate side at an angle $\gamma$ and resonantly couples to the TE-BSW, with a field enhancement $\sim 18$ and $h_{\text {EXC,TE }}=99 \mathrm{~nm}$. Such a configuration permits to excite Dylight650 in its absorption band, at large intensity and in a very thin layer in proximity of the 1DPC surface.

Figure 1d shows a simplified sketch of the fluorescence excitation and detection apparatus used in the experiments. A more detailed description can be found elsewhere [15].

\section{ANGULAR FLUORESCENCE PATTERNS FOR ISOTROPICALLY ORIENTED MOLECULES AT THE SURFACE OF 1DPC}

We consider Dylight650 molecules distributed in a layer much thinner $(1 \mathrm{~nm})$ than $\mathrm{h}_{\mathrm{EXC}, \mathrm{TE}}$, thus the excitation field is uniform. For the moment, we assume that the layer is at $\mathrm{d}=0$ and comment below on the effects arising for $\mathrm{d}>0$ and comparable to $\mathrm{h}_{\mathrm{EM}}$. The dye molecules were modelled as rod-like emitters, 
with the absorption and emission dipole moments aligned along the rod. We also assumed that there is no depolarization during the excitation lifetime and the orientation of the excitation and emission dipoles coincide.

With reference to Figure 2a, for the moment, we describe the isotropically oriented ensemble of dipoles by means of a time-independent normalised distribution function:

$f_{0}(\theta, \varphi)=f_{0}=1 / 4 \pi$

\section{Fluorescence excitation}

The excitation is carried out from the substrate side by means of a CW beam at the angle $\gamma$ in the $\mathrm{xz}$ incidence plane and polarised along the y direction (TE for the 1DPC), as shown in Figure 1d and Figure $2 \mathrm{~b}$. A dipole at $(\theta, \varphi)$ is excited at the rate [2]:

$p_{\text {exc }}(\theta, \varphi) \propto\left|\vec{\mu} \cdot \vec{E}_{\text {exc }}\right|^{2}=\mu^{2} I_{\text {exc }} f_{0} \sin ^{2}(\theta) \sin ^{2}(\varphi)$

where $\vec{\mu}$ is the transition dipole moment. The distribution of emission rates at $\theta$ and $\varphi$ is:

$p_{e m}(\theta, \varphi) \propto \eta p_{e x c} \propto \eta \mu^{2} I_{e x c} f_{0} \sin ^{2}(\theta) \sin ^{2}(\varphi)$

where $\eta$ is the intrinsic fluorescence quantum yield. In the following we assume that $\eta$ is independent from the dipole's orientation and that $\eta=1$.

We represent every emitting dipole as a combination of the three independent $\mathrm{x}, \mathrm{y}$ and $\mathrm{z}$ components, which will be excited at the rates given by the following expressions, obtained by projecting the Eq.(2) along $\mathrm{x}, \mathrm{y}$ and $\mathrm{z}$ :

$$
\left\{\begin{array}{l}
p_{\text {exc }, x}(\vartheta, \varphi)=p_{\text {exc }}(\vartheta, \varphi) \sin ^{2}(\vartheta) \cos ^{2}(\varphi) \\
p_{\text {exc }, y}(\vartheta, \varphi)=p_{\text {exc }}(\vartheta, \varphi) \sin ^{2}(\vartheta) \sin ^{2}(\varphi) \\
p_{\text {exc }, z}(\vartheta, \varphi)=p_{\text {exc }}(\vartheta, \varphi) \cos ^{2}(\vartheta)
\end{array}\right.
$$

The averaged excitation rates for the components are:

$$
\left\{\begin{array}{l}
\bar{p}_{e x c, x} \propto p_{0} \int \sin ^{4}(\vartheta) \sin ^{2}(\varphi) \cos ^{2}(\varphi) \mathrm{d} \Omega=\frac{1}{15} \mu^{2} I_{e x c} \\
\bar{p}_{e x c, y} \propto p_{0} \int \sin ^{4}(\vartheta) \sin ^{4}(\varphi) \mathrm{d} \Omega=\frac{1}{5} \mu^{2} I_{e x c} \\
\bar{p}_{e x c, z} \propto p_{0} \int \sin ^{2}(\vartheta) \cos ^{2}(\vartheta) \sin ^{2}(\varphi) \mathrm{d} \Omega=\frac{1}{15} \mu^{2} I_{e x c}
\end{array}\right.
$$

where $p_{0}=\mu^{2} I_{\text {exc }} f_{0}$. 

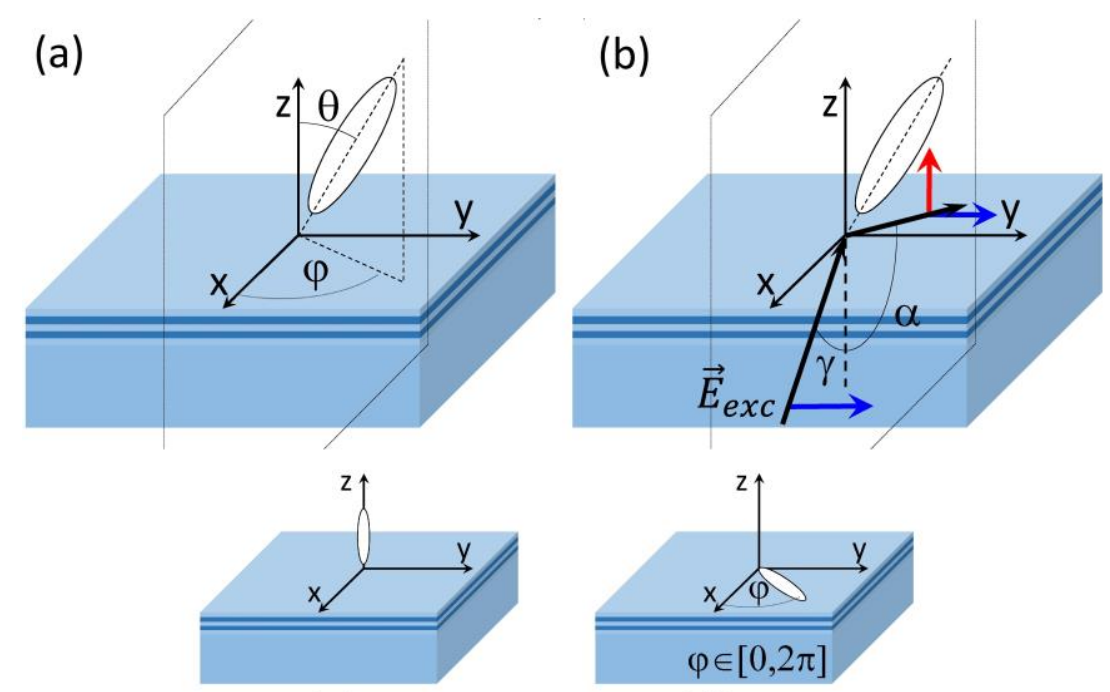

(c)

(d)

Figure 2 - (color online) (a) System of coordinates for the emitter orientation. (b) Fluorescence excitation and collection configuration. (c-d) Base dipole orientations: (c) $\perp$, , d) $\|$.

\section{Fluorescence emission}

Figure $1 \mathrm{~d}$ and Figure $2 \mathrm{~b}$ show that fluorescence is collected from the substrate side in the xz plane at the angle $\alpha$. For the sake of clearness, in Figure $2 b$ we sketched a forward emission configuration, whereas in the real case we use an epi-fluorescence scheme, as in Figure 1d. However, such a difference does not affect our description. Figure $2 \mathrm{~b}$ shows that the emission can be either TE (blue) or TM polarized (red). In the $\mathrm{xy}$ plane, the dipole components along $\mathrm{x}$ and $\mathrm{z}$ can only emit TM radiation, whereas the dipole component along y can only emit TE radiation.

The power emitted by the dipoles is characterized by spectral and angular radiance patterns that were calculated by a theoretical approach describing dipole emitters located inside arbitrary stratified media [22] and based on the Chance, Prock and Silbey theory [23], which applies dyadic Green's functions [24] to describe the effects classically. In the approach, the emission of dipoles in proximity of the 1DPC is addressed by assuming a cylindrical symmetry of their distribution around z. Here, given the TE excitation, such a cylindrical symmetry is lost. However, we can calculate the spectral radiance patterns for dipoles either aligned perpendicularly (Figure 2c) or uniformly distributed in the plane of the stack (Figure 2d) and use the results as a base set to model the emission arising from any specific noncylindrical distribution. 

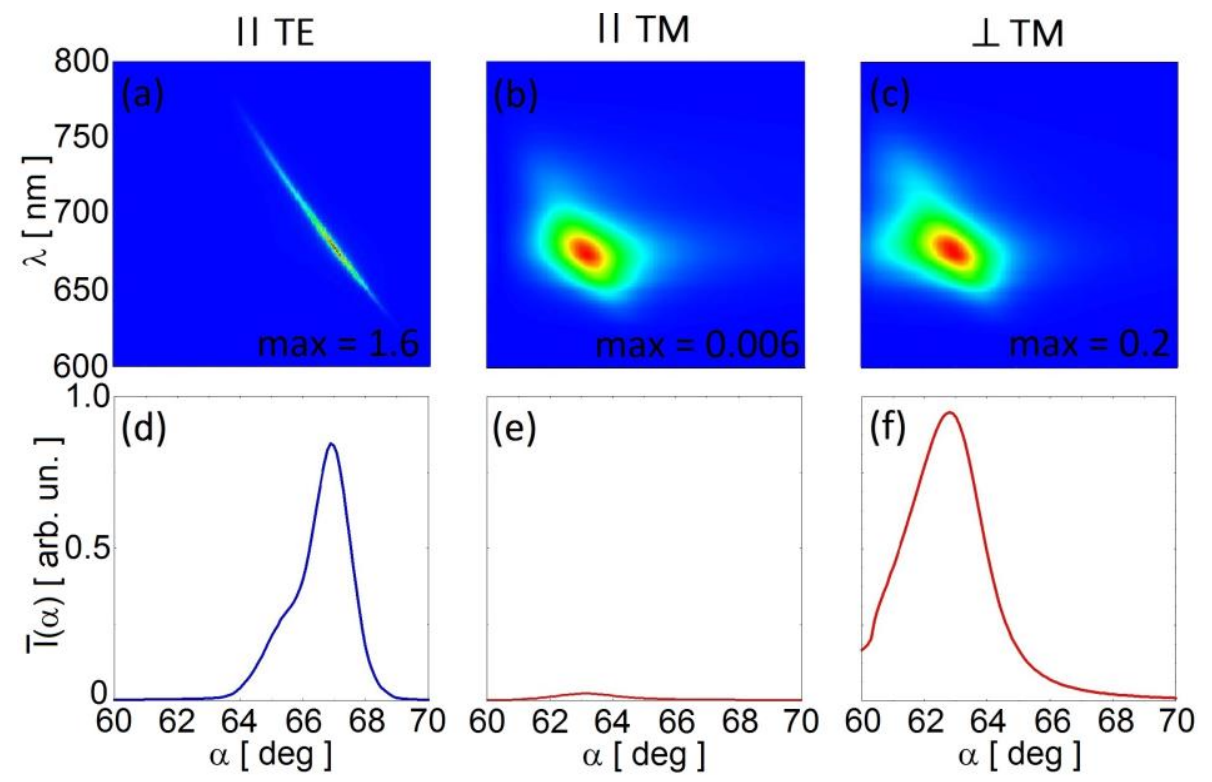

Figure 3 (color online) (a-c) Calculated spectral radiance patterns: (a) $S_{T E}^{\|}(\alpha, \lambda)$, (b) $S_{T M}^{\|}(\alpha, \lambda)$, (c) $S_{T M}^{\perp}(\alpha, \lambda)$. The intensity scale is normalized by the inset factors. (d-f) Radiant intensity of the fluorescence emitted in the substrate. (d) \| TE, (e) \| TM, (f) $\perp$ TM.

In Figure 3a-c we show the spectral radiance patterns $S_{T E}^{\|}(\alpha, \lambda), S_{T M}^{\|}(\alpha, \lambda)$ and $S_{T M}^{\perp}(\alpha, \lambda)$, respectively, for the fluorescent emission in the substrate of dye molecules, calculated for the two base distributions given in Figure 2c-d and for both the TE and the TM polarization.

Integration over $\lambda$ of the spectral radiance patterns yields the radiant intensities $I_{T E}^{\|}(\alpha), I_{T M}^{\|}(\alpha)$ and $I_{T M}^{\perp}(\alpha)$ shown in Figure 3(d-f).

\section{Fluorescence emission under TE excitation}

The TE and TM averaged spectral radiance patterns emitted in the substrate by the isotropically oriented dye labels upon TE excitation are:

$\left\{\begin{array}{l}\bar{S}_{T E}(\alpha, \lambda)=2 \frac{1}{5} S_{T E}^{\|}(\alpha, \lambda) \\ \bar{S}_{T M}(\alpha, \lambda)=\frac{1}{15} S_{T M}^{\perp}(\alpha, \lambda)+2 \frac{1}{15} S_{T M}^{\|}(\alpha, \lambda)\end{array}\right.$

Such expressions were calculated assuming that the average dipolar components $\mathrm{x}, \mathrm{y}, \mathrm{z}$ are excited according to the rates given by the Eq.(5) and that they emit with the spectral radiance patterns $2 S_{T M}^{\|}(\alpha, \lambda)$, $2 S_{T E}^{\|}(\alpha, \lambda)$, and $S_{T M}^{\perp}(\alpha, \lambda)$, respectively. The extra factor 2 in the $\mathrm{x}$ and $\mathrm{y}$ contributions originates from 
the fact that, when calculating $S_{T M}^{\|}(\alpha, \lambda)$ and $S_{T E}^{\|}(\alpha, \lambda)$, we assumed dipoles isotropically distributed in the xy plane; such dipoles will have a component along $\mathrm{x} / \mathrm{y}$ according to the factors $\cos (\varphi) / \sin (\varphi)$ and contribute to the TM / TE spectral radiance with $\cos ^{2}(\varphi) / \sin ^{2}(\varphi)$, which once averaged give the factor 2 . In the second relation in Eq.(6) there is no interference term between the two TM components since, as long as the orientational distribution is symmetric with respect to the excitation field (TE, y), such interference contributions cancel out when averaging over the distribution. Such condition keeps holding also when the distribution is distorted upon photobleaching by the TE polarized excitation field.

The same linear combinatory factors used in Eq.(6) were used to calculate, from the curves in Figure 3(d-f), the TE and TM average radiant intensities $\bar{I}_{T E}(\alpha), \bar{I}_{T M}(\alpha)$ and their sum, shown in Figure 4a. The latter is the observable that is measured in a biosensing assay, in which the angularly dispersed fluorescence emission of dye labels at the surface of the 1DPC is collected by means of a polarization and wavelength insensitive CCD camera [15]. The signal is characterized by the two TE and TM bands, which are peaked at $\alpha_{\mathrm{TE}}$ and $\alpha_{\mathrm{TM}}$ and have amplitudes $\bar{I}_{T E}^{\text {peak }}$ and $\bar{I}_{T M}^{\text {peak }}$. The ratio $R=\bar{I}_{T E}^{\text {peak }} / \bar{I}_{T M}^{\text {peak }}$ can be used to probe in experiments the orientational distribution of the emitters. [15]
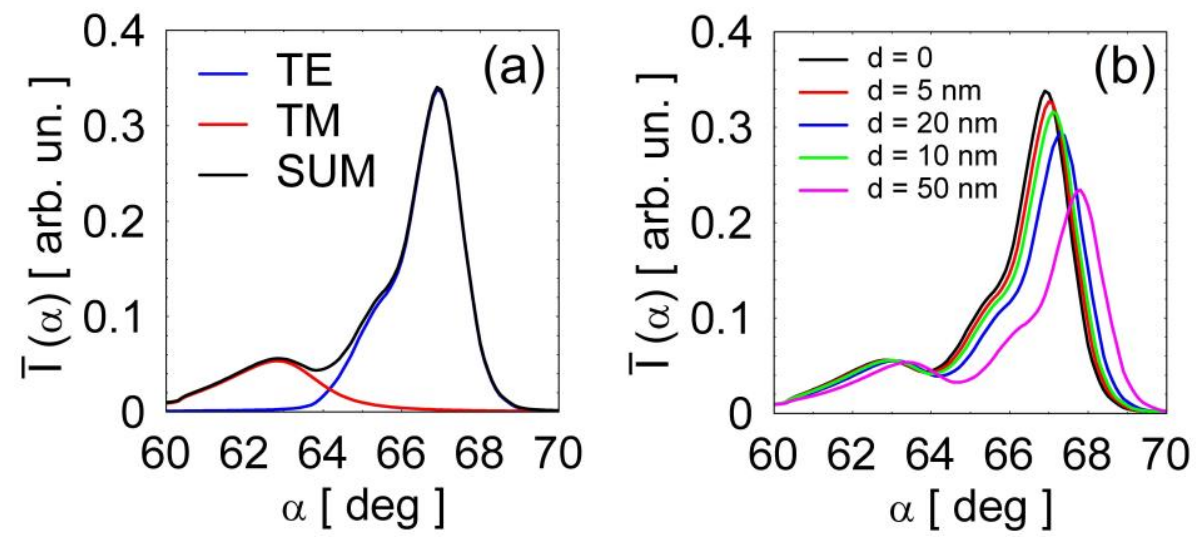

Figure 4 - (color online) (a) Average radiant intensities. (b) Average radiant intensities for different values of d.

From Figure $4 \mathrm{a}$ we found that, for isotropically angularly distributed emitters, $R_{0}=6.1$. Such a value depends on the specific 1DPC design and dye spectrum and on the distance $d$. It is of particular relevance the dependency on $d$, since it can vary much in the real experimental case, due to the presence of any capture or blocking biomolecular spacer, as sketched in Figure 1 [15]. Figure 4b shows the $\bar{I}(\alpha)$ plots calculated for different thicknesses $d$ of a spacer layer with refractive index $\mathrm{n}_{\mathrm{BIO}}=1.4$. Coupling of the fluorescent emission to the TE-BSW decreases remarkably when increasing d, whereas the TM 
contribution is almost constant, as expected due to its much weaker localization. The ratio $R_{0}$ decreases linearly with a slope $\partial R_{0} / \partial d \sim-0.036 \mathrm{~nm}^{-1}$, passing from $R_{0}(d=0 \mathrm{~nm})=6.1$ to $R_{0}(d=50 \mathrm{~nm})=$ 4.2. In the calculations, we took into account that the spacer affects also the local intensity of the excitation field, which however changes by less than $1 \%$. The angular shift of the emission in Figure $4 \mathrm{~b}$ is instead due to the perturbation of the BSW dispersion introduced by the spacer.

\section{EFFECT OF PHOTOBLEACHING}

We consider now that the fluorescent molecules at the 1DPC surface can be photobleached when illuminated in the absorption band. Such a situation is much likely since the labels are excited at resonance and the TE-BSW field is very intense. The dye labels will be photobleached mainly along the y direction. For the moment let's assume that the orientation of the molecules cannot change along time. In the following section we shall address the case in which depolarization due to rotational diffusion also takes place.

Under CW illumination the PB rate is proportional [25] to the excitation probability given by the Eq.(2) and it depends on the relative orientation between the excitation field (y) and the molecule, through the factor $\sin ^{2}(\theta) \sin ^{2}(\varphi)$. The rate equation for the PB process is then given by:

$\frac{\partial f(\vartheta, \varphi, t)}{\partial t}=-\sigma I_{e x c} f(\vartheta, \varphi, t) \sin ^{2}(\vartheta) \sin ^{2}(\varphi)$

where $\sigma$ is the $\mathrm{PB}$ cross section and $f(\vartheta, \varphi, t)$ is time dependent and not anymore normalised to unity. Assuming that at $\mathrm{t}=0$ the molecules are isotropically oriented with $f(\vartheta, \varphi, 0)=f_{0}=1 / 4 \pi$, the solution of the Eq.(7) is:

$f(\vartheta, \varphi, t)=\frac{1}{4 \pi} e^{-\sigma I_{e x c} \sin ^{2}(\vartheta) \sin ^{2}(\varphi) t}$

Therefore, under excitation and PB by a TE polarized field, the dipole's excitation probability is given by:

$p_{\text {exc }}(\vartheta, \varphi) \propto \mu^{2} I_{e x c} f(\vartheta, \varphi, t) \sin ^{2}(\vartheta) \sin ^{2}(\varphi)=\frac{\mu^{2} I_{e x c}}{4 \pi} e^{-\sigma I_{e x c} \sin ^{2}(\vartheta) \sin ^{2}(\varphi) t} \sin ^{2}(\vartheta) \sin ^{2}(\varphi)$

The average excitation rates of the $\mathrm{x}, \mathrm{y}, \mathrm{z}$ dipoles components in the case of bleaching are:

$\left\{\begin{array}{l}\bar{p}_{\text {exc }, x}(t) \propto \mu^{2} I_{\text {exc }} \int f(\vartheta, \varphi, t) \sin ^{4}(\vartheta) \sin ^{2}(\varphi) \cos ^{2}(\varphi) \mathrm{d} \Omega \\ \bar{p}_{\text {exc }, y}(t) \propto \mu^{2} I_{\text {exc }} \int f(\vartheta, \varphi, t) \sin ^{4}(\vartheta) \sin ^{4}(\varphi) \mathrm{d} \Omega \\ \bar{p}_{\text {exc }, z}(t) \propto \mu^{2} I_{\text {exc }} \int f(\vartheta, \varphi, t) \sin ^{2}(\vartheta) \cos ^{2}(\vartheta) \sin ^{2}(\varphi) \mathrm{d} \Omega\end{array}\right.$

The integrals in the Eq.(10) can be analytically calculated, as reported in the Supplementary Material. We 
find:

$\left\{\begin{array}{l}\bar{p}_{\text {exc }, x}(t) \propto \mu^{2} I_{\text {exc }} \cdot F_{1}(t) \\ \bar{p}_{\text {exc }, y}(t) \propto \mu^{2} I_{\text {exc }} \cdot F_{2}(t) \\ \bar{p}_{\text {exc }, z}(t) \propto \mu^{2} I_{\text {exc }} \cdot F_{1}(t)\end{array}\right.$

with:

$F_{1}(t)=\frac{\sqrt{\pi} \cdot \operatorname{erf}\left(\sqrt{\sigma I_{\text {exc }} t}\right) \cdot\left[2 \sigma I_{\text {exc }} t-3\right]+6 \mathrm{e}^{-\sigma I_{\text {exc }} t} \sqrt{\sigma I_{\text {exc }} t}}{16\left(\sigma I_{\text {exc }}\right)^{5 / 2} t^{5 / 2}}$

$F_{2}(t)=\frac{\mathrm{e}^{-\sigma I_{\text {exc }} t}\left[-6 \sqrt{\sigma I_{\text {exc }} t}-4\left(\sigma I_{\text {exc }}\right)^{3 / 2} t^{3 / 2}+3 \mathrm{e}^{\sigma I_{\text {exc }} t} \sqrt{\pi} \operatorname{erf}\left(\sqrt{\sigma I_{\text {exc }} t}\right)\right]}{8\left(\sigma I_{\text {exc }}\right)^{5 / 2} t^{5 / 2}}$

The average spectral radiance and radiant intensity under photobleaching can be calculated with expressions similar to the Eq.(6), where now the weighing factors are $F_{1}(t), F_{2}(t)$ and $F_{1}(t)$, for the $\mathrm{x}, \mathrm{y}$ and $\mathrm{z}$ components, respectively. In particular for the average total radiant intensity emitted in the substrate we get:

$\bar{I}(\alpha, t)=2 F_{2}(t) \cdot I_{T E}^{\|}(\alpha)+F_{1}(t)\left[I_{T M}^{\perp}(\alpha)+2 I_{T M}^{\|}(\alpha)\right]$

In Figure 5a we show plots of $\bar{I}(\alpha, t)$ calculated at several different normalized times $\sigma I_{\text {exc }} t$ along the illumination. Clearly the ratio of the amplitudes of the two main peaks at $\alpha_{\mathrm{TE}}$ and $\alpha_{\mathrm{TM}}$ changes during the photobleaching process.

Following from the Eq.(13), the ratio $\mathrm{R}(\mathrm{t})$ of the peak intensities is given by:

$$
\begin{aligned}
& R(t)=\frac{\bar{I}\left(\alpha_{T E}, t\right)}{\bar{I}\left(\alpha_{T M}, t\right)}=\frac{2 F_{2}(t) \cdot I_{E}^{\|}\left(\alpha_{T E}, \lambda\right)+F_{1}(t)\left[I_{T M}^{\perp}\left(\alpha_{T E}, \lambda\right)+2 I_{T M}^{I}\left(\alpha_{T E}, \lambda\right)\right]}{2 F_{2}(t) \cdot I_{T E}^{I}\left(\alpha_{T M}, \lambda\right)+F_{1}(t)\left[I_{T M}^{\perp}\left(\alpha_{T M}, \lambda\right)+2 I I_{T M}^{\|}\left(\alpha_{T M}, \lambda\right)\right]}= \\
& =\frac{F_{2}(t) \cdot 1.70+F_{1}(t) \cdot 0.036}{F_{2}(t) \cdot 0.014+F_{1}(t) \cdot 0.80}
\end{aligned}
$$

where the numerical coefficients were extracted from the data plotted in Figure 3(d-f). R(t) takes the value $\mathrm{R}_{0}=6.1$ at $\mathrm{t}=0$, decreases as a function of time according to the log-log plot shown in Figure $5 \mathrm{~b}$, and approaches a hyperbolic behaviour for large times. 

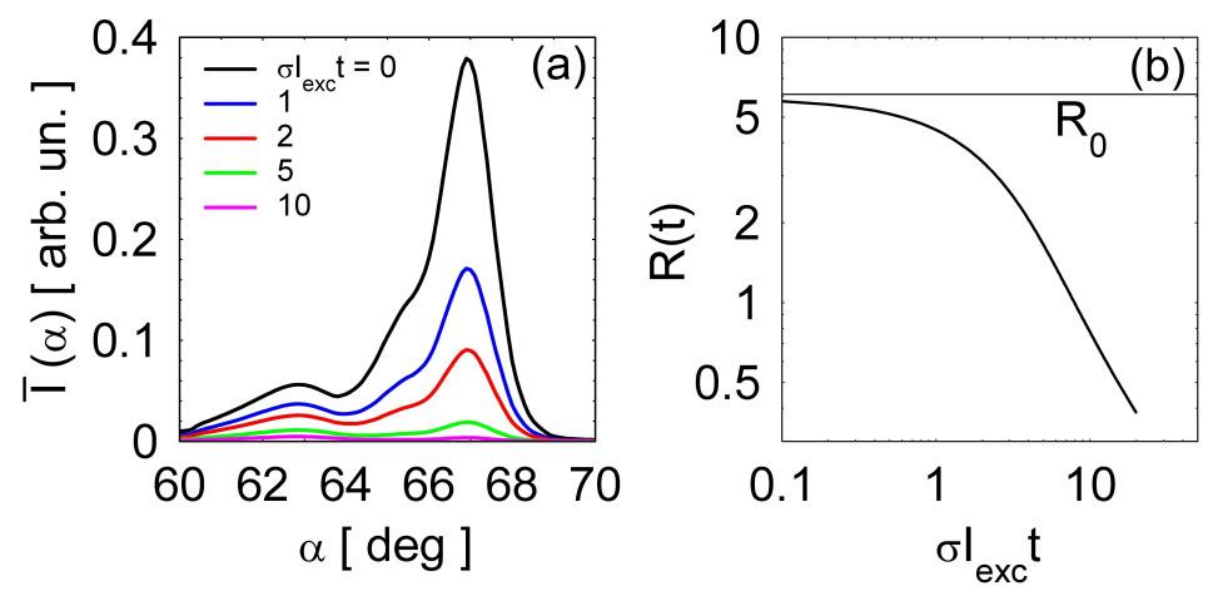

Figure 5 - (color online) (a) Total average radiant intensity under photobleaching conditions, calculated for several different values of $\sigma I_{\text {exc }} t$. (b) Time dependence of $\mathrm{R}(\mathrm{t})$ as a function of $\sigma I_{\text {exc }} t$.

\section{COMPLETE APPROACH WITH PHOTOBLEACHING AND ROTATIONAL DIFFUSION}

The results obtained above confirm our experimental observation [15] that photobleaching modifies the orientational distribution of the emitters from an isotropic to a cylindrical symmetry around the TE direction. However, the experiments also show that rotational diffusion cannot be neglected. Depending on the strength of their bond to the 1DPC surface, the molecules can show depolarization kinetics which span from a slow regime, molecules tightly bound at the surface, to a fast regime for free molecules, with all intermediate cases. Above we treated the case of tightly bound molecules. Usually in experiments that deal with fluorescence emission at the surface of a 1 DPC $[8,12,13,20]$, it is assumed that the orientational distribution of the emitters is isotropic, independently from the excitation polarization, due to depolarization in the excited state [14]. This is the extreme case of completely free molecules. Our experimental results [15] show that often we are in an intermediate condition, imposing to address depolarization in the frame of a more detailed model.

Combining the well-known Smoluchowsky-Einstein equation [26] for rotational diffusion of rod-like molecules with the Eq.(7) for PB we get the following equation:

$\frac{\partial f(\theta, \varphi, t)}{\partial t}=\frac{k_{B} T}{\zeta} \frac{1}{\sin \theta} \frac{\partial}{\partial \theta}\left(\sin \theta \frac{\partial f(\theta, \varphi, t)}{\partial \theta}\right)+\frac{k_{B} T}{\zeta} \frac{1}{\sin ^{2} \theta} \frac{\partial^{2} f(\theta, \varphi, t)}{\partial \varphi^{2}}-\sigma I_{e x c} f(\theta, \varphi, t) \sin ^{2} \theta \sin ^{2} \varphi$

where $k_{B} T$ is the thermal energy and $\zeta$ is a friction coefficient between the molecule and the host medium [26].

The Eq.(15) can be solved by expanding $f(\theta, \varphi, t)$ over the Laplace spherical harmonics $Y_{l}^{m}(\theta, \varphi)$, 
which are solutions of the rotational diffusion equation [26]:

$f(\theta, \varphi, t)=\sum_{l=0}^{\infty} \sum_{m=-l}^{m=l} A_{l}^{m}(t) Y_{l}^{m}(\theta, \varphi)$

where:

$Y_{l}^{m}(\theta, \varphi)=a_{l m} e^{i m \varphi} P_{l}^{m}(\cos \theta)$

$a_{l m}=\frac{1}{\sqrt{2 \pi}}(-1)^{m} \sqrt{\frac{(2 l+1)(l-m) !}{2(l+m) !}}$

and $P_{l}^{m}(\cos \theta)$ are the associated Legendre polynomials [27].

Under this approach the Eq.(15), as described in detail in the Supplementary Material, reduces to a set of coupled differential equations for the time dependent $A_{l}^{m}(t)$ :

$\frac{d \boldsymbol{A}(t)}{d t}=\boldsymbol{H} \cdot \boldsymbol{A}(t)=\left(\boldsymbol{H}^{(\boldsymbol{P B})}+\boldsymbol{H}^{(\boldsymbol{D E P})}\right) \cdot \boldsymbol{A}(t)$

where:

$\boldsymbol{A}(t)=\left(A_{0}^{0}(t), A_{1}^{-1}(t), A_{1}^{0}(t), A_{1}^{1}(t), \ldots, A_{N}^{N}(t)\right)$

$\mathrm{N}$ is the maximum order of the expansion and the matrix operators $\boldsymbol{H}^{(\boldsymbol{P B})}$ and $\boldsymbol{H}^{(\boldsymbol{D E P})}$, whose complete expressions are given in the Supplementary Material, govern PB and depolarization, respectively.

The solution of the Eq.(19) is:

$\boldsymbol{A}(t)=C_{1} e^{\lambda_{1} t} \boldsymbol{u}_{\mathbf{1}}+C_{2} e^{\lambda_{2} t} \boldsymbol{u}_{2} \ldots+C_{M} e^{\lambda_{M} t} \boldsymbol{u}_{\boldsymbol{M}}$

where $\lambda_{i}$ and $\boldsymbol{u}_{\boldsymbol{i}}$ are the eigenvalues and eigenvectors of $\boldsymbol{H}$ and the constants $C_{i}$ depend on the initial conditions $A_{l}^{m}(0)$.

Once the $A_{l}^{m}(t)$ are found, we calculate $f(\theta, \varphi, t)$ by the Eq.(16) and determine analytically the average excitation probabilities, as described in the Supplementary Material:

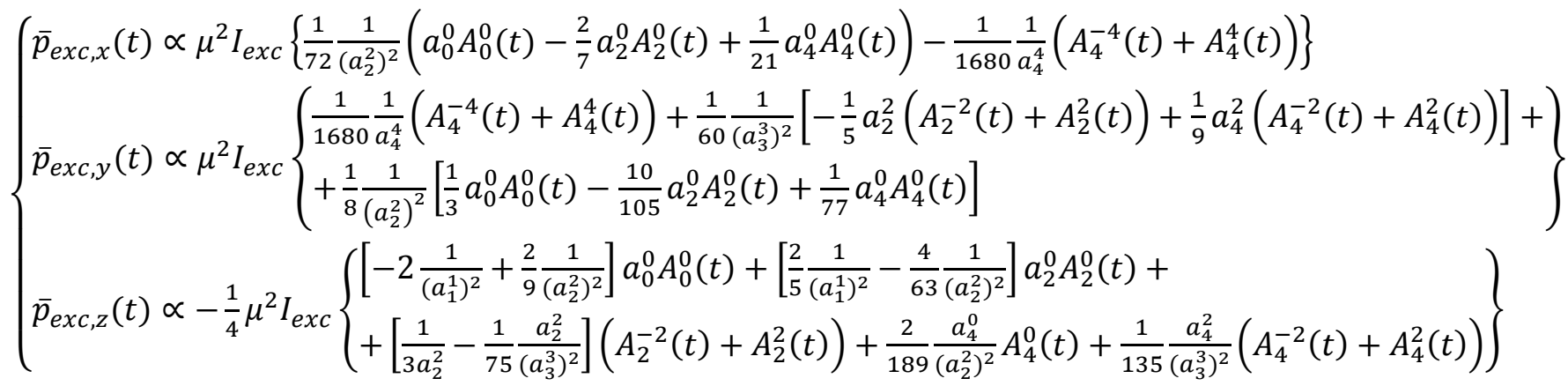


where the even $A_{l}^{m}(t)$ up to the order $l=4$ are involved.

The total radiant intensity can be calculated as in the case without depolarization given in the Eq.(14), where now the weighing factors are given by the Eq.(22), normalized to the $\mu^{2} I_{\text {exc }}$ pre-factor :

$\bar{I}(\alpha, t)=\frac{1}{\mu^{2} I_{\text {exc }}}\left[2 \bar{p}_{\text {exc }, y}(t) \cdot I_{T E}^{\|}(\alpha)+\bar{p}_{\text {exc }, z}(t) \cdot I_{T M}^{\perp}(\alpha)+2 \bar{p}_{\text {exc }, x}(t) \cdot I_{T M}^{\|}(\alpha)\right]$

The ratio $R(t)$ is then given by:

$R(t)=\frac{2 \bar{p}_{\text {exc }, y}(t) \cdot 0.84+\bar{p}_{\text {exc }, z}(t) \cdot 0.031+2 \bar{p}_{\text {exc }, x}(t) \cdot 0.0025}{2 \bar{p}_{\text {exc }, y}(t) \cdot 0.0068+\bar{p}_{\text {exc }, z}(t) \cdot 0.76+2 \bar{p}_{\text {exc }, x}(t) \cdot 0.021}$

where the numerical values for $I_{T E, T M}^{\|, \perp}\left(\alpha_{T E, T M}\right)$ were taken from Figure $3 \mathrm{~d}-\mathrm{f}$.

\section{DISCUSSION}

In the present section, first of all we discuss the characteristics of the solutions of the Eq.(15) for different initial conditions $f(\theta, \varphi, 0)$, with the aim to analyse a set of standard solutions that can be compared to experiments [15].

In a second part, we discuss the consequences of anisotropic PB on fluorescence based biosensing assays and propose some experimental procedures to rule out $\mathrm{PB}$, which is of paramount importance when targeting quantitative and reliable detection at small fluorophores' concentrations.

In a third part, we propose to exploit anisotropic PB to investigate depolarization kinetics of biomolecules bound at a surface, similar to what is done with fluorescence recovery after PB (FRAP) [2] to investigate their spatial diffusion.

\section{Photobleaching and orientational diffusion under different initial conditions}

The Laplace harmonics expansion permits to study the kinetics of the distributions with an arbitrary initial condition. Figure 6 shows the temporal evolution of $f(\theta, \varphi, t)$, calculated for the values $\sigma I_{\text {exc }}=0.07 s^{-1}$ and $k_{B} T / \zeta=0.003 s^{-1}$, which are typical in experiments. The distributions were calculated for the three specific initial conditions indicated in Figure 6 (isotropic, laying and standing). The small value of $k_{B} T / \zeta$ hinders rotational diffusion and makes that angular hole burning can take place along $\mathrm{y}$. Meanwhile, PB occurs and the overall amplitude of $f(\theta, \varphi, t)$ decreases. In all cases, diffusion makes that the symmetry of the distributions converges towards that found for the isotropic case. Results obtained for different $\sigma I_{\text {exc }}$ and $k_{B} T / \zeta$ can be found in the Supplementary Material.

The results can be analysed in a better way by comparing the temporal dependency of $\mathrm{R}(\mathrm{t})$. Figure $7 \mathrm{a}$ 
shows, for the isotropic case, the calculated $\mathrm{R}(\mathrm{t})$ for $k_{B} T / \zeta=0.003 \mathrm{~s}^{-1}$ and for several different values of $\sigma I_{\text {exc }}$. Increasing $\sigma I_{\text {exc }}$ leads to a faster decay of $\mathrm{R}(\mathrm{t})$, which always reaches a plateau value that is determined by the balance between rotational diffusion and photobleaching.

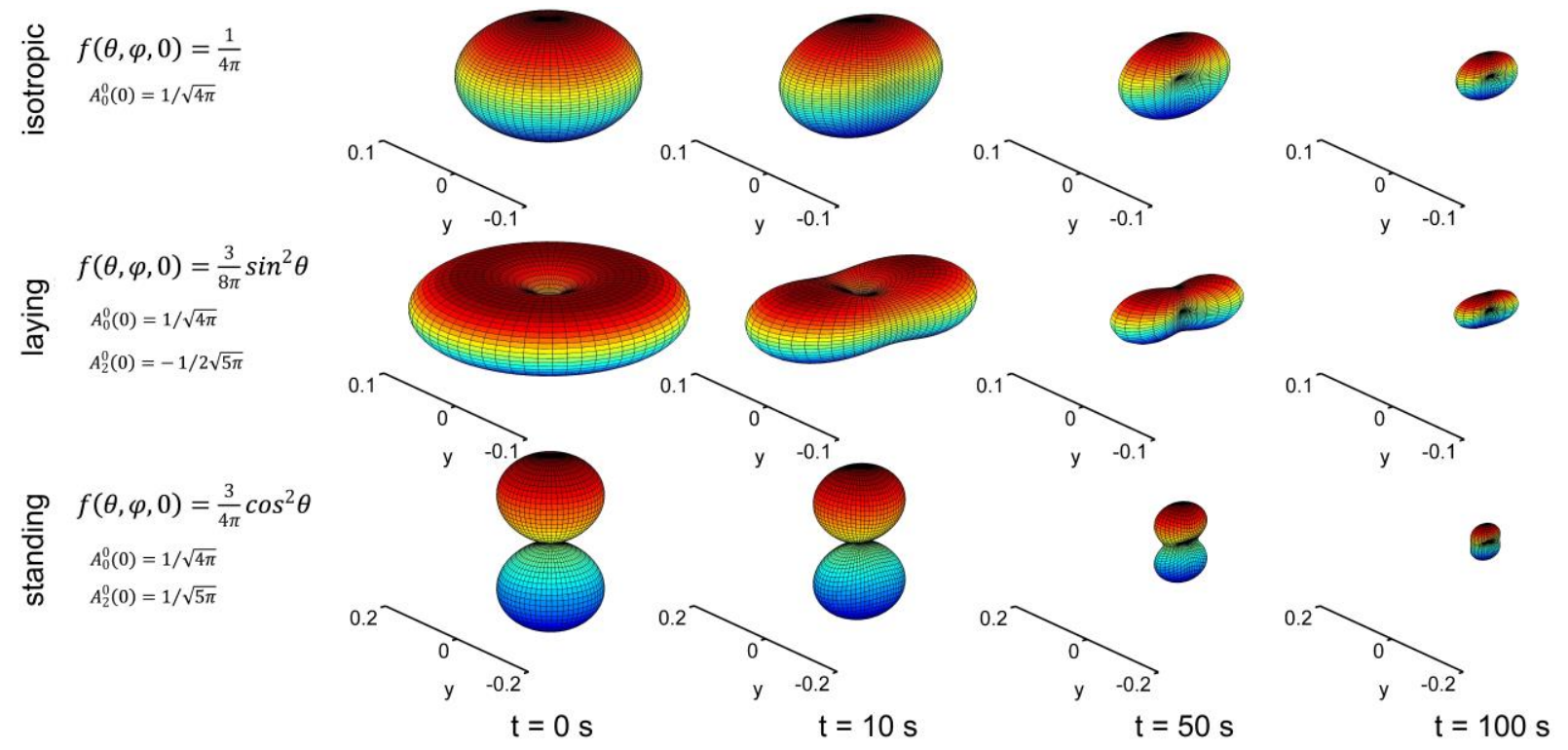

Figure 6 - (color online) Temporal evolution of $f(\theta, \varphi, t)$ with: (top) isotropic, (middle) laying or (bottom) standing (bottom) initial conditions, for $\sigma I_{\text {exc }}=0.07 \mathrm{~s}^{-1}$ and $k_{B} T / \zeta=0.003 \mathrm{~s}^{-1}$. Note the change of the scale in the bottom case. AVI videos of the simulations are available in the Supplementary Material.

Figure $7 \mathrm{~b}$ shows the $\mathrm{R}(\mathrm{t})$ plots for the isotropic, laying and standing cases extracted from Figure 6. Clearly, different initial conditions give rise to dramatic changes of the kinetics, which span from a growth to a decay regime. In the decay regime the time constant is strongly modified. In all cases, $R(t)$ reaches the same equilibrium value fixed by the balance between photobleaching and orientational diffusion.
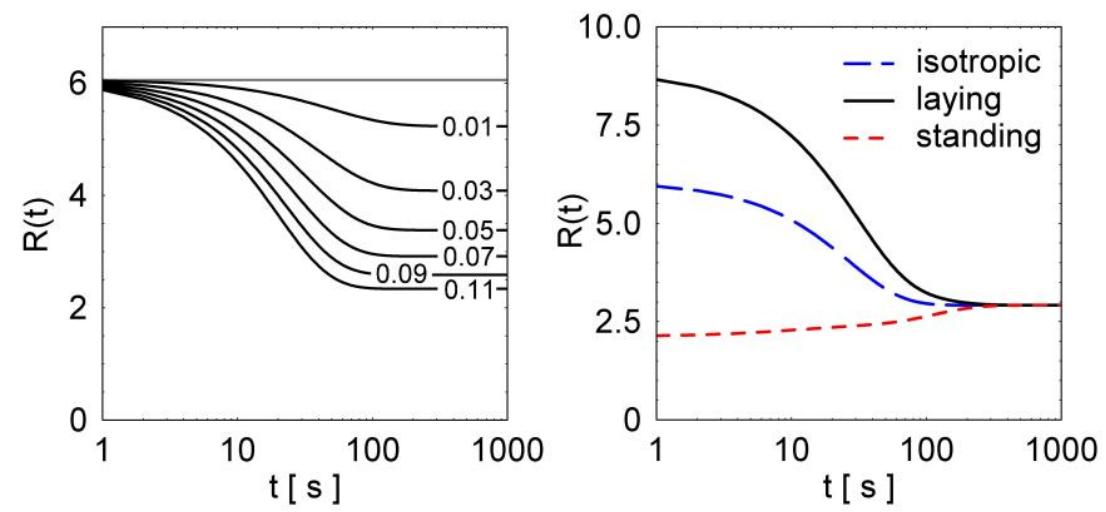

Figure 7 - (color online) (a) $\mathrm{R}(\mathrm{t})$ calculations for $k T / \zeta=0.003 \mathrm{~s}^{-1}$ and for several different values of $\sigma I_{\text {exc }}$. (b) $\mathrm{R}(\mathrm{t})$ calculations for $k T / \zeta=0.003 s^{-1}$ and $\sigma I_{e x c}=0.07 s^{-1}$ and for three different initial conditions. 


\section{Managing photobleaching in fluorescence biosensing experiments}

From the analysis above, it is clear that PB cannot be neglected when making use of fluorescent labels in biological assay formats in proximity of 1DPC sustaining BSW and in general of highly resonant photonic structures. On one hand, photobleaching breaks the assumption of linear proportionality between the fluorescence intensity and the surface density of labelled biomolecules captured at the surface. On the other hand, energy redistribution between the TE and TM polarized emission channels might generate distortions when monitoring just one polarization.

One possibility to manage PB is to collect the average radiant intensity, shown in Figure 4a, at fixed time intervals $\Delta \mathrm{t}$ under the same resonant excitation conditions [15]. The TE and TM peak intensities $I_{T E}^{\text {peak }}$ and $I_{T M}^{\text {peak }}$ may display multi-exponential decays, which can be fitted separately by the Eq. (24) and traced back to $\Delta \mathrm{t}=0$ to get the bleaching free signal. Furthermore, monitoring the ratio $\mathrm{R}_{\Delta \mathrm{t}}$ as a function of $\Delta \mathrm{t}$, permits to verify that the signals are properly acquired, since we know that $\mathrm{R}_{\Delta \mathrm{t}}$ extrapolates to $R_{0}=$ 6.1 for fluorophores at the surface of the 1DPC and for an initially isotropic distribution of the emitters. However, we should keep in mind that $R_{0}$ might depend on the specific 1DPC design (Figure 1), on the distance of the emitters from the surface (Figure 4b) and on the initial distribution of emitters (Figure 7b). The latter effect is not expected, since usually biomolecules are randomly labelled with more than one fluorophore (2 to 4) [28], but could be exploited in future applications upon careful control of the labelling process.

\section{Fluorescence recovery after orientational photobleaching (FROP)}

The possibility to obtain in a single measurement both the TE and TM components of the resonant fluorescence signal of labelled biomolecules bound at a surface paves the way towards accessing to their orientation and to their depolarization kinetics under non stationary conditions. Such type of measurement is not possible with conventional fluorescence anisotropy techniques [2]. The use of other types of surface electromagnetic waves, such as SPP, is also not possible since they are only TM polarized [21].

Figure 8a shows a fluorescence recovery after orientational photobleaching (FROP) virtual experiment

in which the normalized intensities $I_{T E}^{\text {peak }} / I_{\text {exc }}$ and $I_{T M}^{\text {peak }} / I_{\text {exc }}$ are plotted as a function of time, for isotropically oriented fluorophores at the 1DPC surface and $k_{B} T / \zeta=0.003 \mathrm{~s}^{-1}$. 
In a first PB dominated phase up to $t_{\mathrm{pb}}=25 \mathrm{~s}$ (white background), upon TE-BSW excitation at large intensity $\sigma I_{\text {exc }}=0.07 s^{-1}, f(\theta, \varphi, t)$ is driven off equilibrium and the TE (blue) and TM (red) signals decrease. In the following depolarization phase from $\mathrm{t}_{\mathrm{pb}}$ to $\mathrm{t}_{\mathrm{pb}}+600 \mathrm{~s}$ (grey background), the excitation intensity is decreased in order to change the balance between the PB and depolarization rates and a recovery of the fluorescence signals is observed. Depending on $\sigma I_{\text {exc }}$, we observe different recovery kinetics. For negligible PB ( $\sigma I_{\text {exc }}=10^{-7} s^{-1}$, solid), the signals partially recover while the orientational distribution relaxes towards isotropy. For larger $\sigma I_{\text {exc }}\left(7 \cdot 10^{-4} s^{-1}\right.$, dashed; $7 \cdot 10^{-3} s^{-1}$, dadot), the residual PB competes with depolarization and the curves show maxima before decreasing monotonically, while isotropy is partially recovered.

FROP can be better monitored if one monitors $R(t)$, as shown in Figure $8 b$, for the cases $t_{p b}=25 \mathrm{~s}$ (black) and $t_{\mathrm{pb}}=100 \mathrm{~s}(\mathrm{red})$. During the PB phase, $\mathrm{R}(\mathrm{t})$ decreases starting from $\mathrm{R}_{0}$ and during the recovery phase relaxes back to an equilibrium plateau. The plateau is at $\mathrm{R}_{0}$ if $\mathrm{PB}$ can be neglected during recovery (solid curves), otherwise it is smaller depending on the relative weight of the PB and relaxation rates (dashed curves). If the PB phase is extended to $100 \mathrm{~s}$, the system is driven more off equilibrium, nevertheless $\mathrm{R}(\mathrm{t})$ recovers to the same plateaus. Such last result puts into evidence the powerfulness of the $\mathrm{R}(\mathrm{t})$ analysis, which is ratiometric and therefore independent on the number of active emitters [2].

For negligible PB, FROP is governed by the Eq.(19) with $\boldsymbol{H}^{(\boldsymbol{P B})}=0$ :

$$
\frac{d \boldsymbol{A}(t)}{d t}=\boldsymbol{H}^{(\boldsymbol{D E P})} \cdot \boldsymbol{A}(t)
$$

Since $\boldsymbol{H}^{(\boldsymbol{D E P})}$ is diagonal (see Supplementary Material):

$$
\boldsymbol{H}_{l(l+1)+m, l(l+1)+m}^{(D E P)}=-k_{B} T / \varsigma l(l+1)=-D_{l}
$$

we get:

$A_{l}^{m}(t)=A_{l}^{m}\left(t_{p b}\right) e^{-D_{l}\left(t-t_{p b}\right)}$

Since only $A_{2}^{m}(t)$ and $A_{4}^{m}(t)$ are non-zero, the recovery of $\mathrm{R}(\mathrm{t})$, given by the Eq.(24), is governed just by $D_{2}=6 k_{B} T / \zeta$ and $D_{4}=20 k_{B} T / \zeta$. However, for a limited PB dose and small $f(\theta, \varphi, t)$ distortions, which is true for both cases shown in Figure $8 \mathrm{~b}, A_{4}^{m}(t)$ can be neglected within a few percent error, and $\mathrm{R}(\mathrm{t})$ is well described by a single exponential with a decay constant $\tau_{r e c}=1 / D_{2}$, as shown in Figure $8 \mathrm{~b}$. Such last result suggests that by performing FROP experiments and monitoring the recovery of the ratiometric quantity $\mathrm{R}(\mathrm{t})$ one can directly access to the rotational diffusion coefficient of any fluorescent molecule 
bound in proximity of the 1DPC surface with the resolution provided by the BSW evanescent tail.
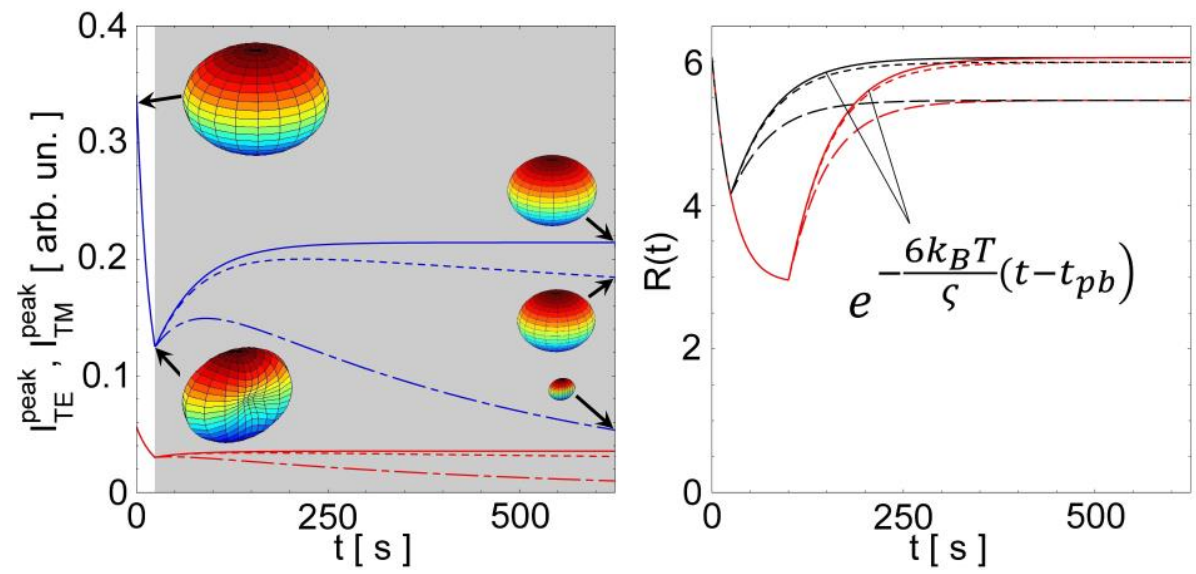

Figure 8 - (a) Calculated FROP normalized peak radiant intensities: (blue) $I_{T E}^{\text {peak }} / I_{\text {exc }}$, (red) $I_{T M}^{\text {peak }} / I_{\text {exc }}$. (b) R(t) during the PB and FROP phase.

\section{CONCLUSIONS}

We derived a theoretical model describing the interplay of PB and rotational diffusion of organic emitters bound at the surface of a 1DPC fluorescence biosensors sustaining BSW. The results of the simulations show that PB can severely affect the fluorescence emission in terms of total intensity and angular distribution. Such result indicates that a careful analysis of fluorescence anisotropy in biosensing experiments with PC must be carried out. We applied the model to describe some case study conditions, which can take place in the real experiments, and proposed a procedure to rule out the detrimental contribution of photobleaching to the biosensors' response. Finally, we proposed a procedure based on fluorescence recovery after orientational photobleaching to study rotational diffusion of dye-labelled biomolecules bound at a surface.

\section{ACKNOWLEDGEMENTS}

The authors wish to thank A. Sinibaldi, N. Danz and A. Occhicone for fruitful discussions. This work was funded by the European Commission (BILOBA, 318035) and by the Regione Lazio (TURNOFF, 852017-14945). 


\section{REFERENCES}

1. A. P. Demchenko, Introduction to Fluorescence Sensing, 2nd ed. (Springer Nature Switzerland AG, 2009).

2. J. R. Lakowicz, Principles of Fluorescence Spectroscopy, Third Edit (Springer US, 2006).

3. K. Toma, M. Vala, P. Adam, J. Homola, W. Knoll, and J. Dostálek, "Compact surface plasmonenhanced fluorescence biochip," Opt. Express 21, 10121 (2013).

4. P. C. Mathias, N. Ganesh, L. L. Chan, and B. T. Cunningham, "Combined enhanced fluorescence and label-free biomolecular detection with a photonic crystal surface," Appl. Opt. 46, 2351 (2007).

5. N. Vats, S. John, and K. Busch, "Theory of fluorescence in photonic crystals," Phys. Rev. A 65, $1-13$ (2002).

6. V. Chaudhery, M. Lu, C. S. Huang, S. George, and B. T. Cunningham, "Photobleaching on Photonic Crystal Enhanced Fluorescence Surfaces," J. Fluoresc. 21, 707-714 (2011).

7. A. Sinibaldi, A. Fieramosca, R. Rizzo, A. Anopchenko, N. Danz, P. Munzert, C. Magistris, C. Barolo, and F. Michelotti, "Combining label-free and fluorescence operation of Bloch surface wave optical sensors," Opt. Lett. 39, 2947-2950 (2014).

8. K. Toma, E. Descrovi, M. Toma, M. Ballarini, P. Mandracci, F. Giorgis, A. Mateescu, U. Jonas, W. Knoll, and J. Dostálek, "Bloch surface wave-enhanced fluorescence biosensor," Biosens. Bioelectron. 43, 108-114 (2013).

9. P. Yeh, A. Yariv, and C.-S. Hong, "Electromagnetic propagation in periodic stratified media I General theory*," J. Opt. Soc. Am. 67, 423-438 (1977).

10. A. Sinibaldi, A. Fieramosca, N. Danz, P. Munzert, A. Occhicone, C. Barolo, and F. Michelotti, "Effects of Reabsorption due to Surface Concentration in Highly Resonant Photonic Crystal Fluorescence Biosensors," J. Phys. Chem. C 122, 26281-26287 (2018).

11. A. Angelini, E. Barakat, P. Munzert, L. Boarino, N. De Leo, E. Enrico, F. Giorgis, H. P. Herzig, C. F. Pirri, and E. Descrovi, "Focusing and Extraction of Light mediated by Bloch Surface Waves," Sci. Rep. 4, 5428 (2014).

12. S. D. Choudhury, R. Badugu, and J. R. Lakowicz, "Directing Fluorescence with Plasmonic and Photonic Structures," Accounts Chem. Res. 48, 2171-2180 (2015).

13. R. Rizzo, M. Alvaro, N. Danz, L. Napione, E. Descrovi, S. Schmieder, A. Sinibaldi, R. 
Chandrawati, S. Rana, P. Munzert, T. Schubert, E. Maillart, A. Anopchenko, P. Rivolo, A. Mascioletti, F. Sonntag, M. M. Stevens, F. Bussolino, and F. Michelotti, "Bloch surface wave label-free and fluorescence platform for the detection of VEGF biomarker in biological matrices," Sensors Actuators, B Chem. 255, 2143-2150 (2018).

14. F. Perrin, "Polarisation de la lumière de fluorescence . Vie moyenne des molécules dans 1 ' etat excité," 7, 390-401 (1926).

15. E. Sepe, A. Sinibaldi, N. Danz, P. Munzert, and F. Michelotti, "Anisotropic Fluorescence Emission and Photobleaching of Fluorophores in Proximity of One Dimensional Photonic Crystals sustaining Bloch Surface Waves. II. Experiments," J. Phys. Chem. C (n.d.).

16. R. Rizzo, N. Danz, F. Michelotti, E. Maillart, A. Anopchenko, and C. Wächter, "Optimization of angularly resolved Bloch surface wave biosensors," Opt. Express 22, (2014).

17. "https://topas.com/," .

18. P. Munzert, N. Danz, A. Sinibaldi, and F. Michelotti, "Multilayer coatings for Bloch surface wave optical biosensors," Surf. Coat. Technol. 314, 79-84 (2017).

19. K. H. Drexhage, "Influence of a dielectric interface on fluorescence decay time," J. Lumin. 1,2, 693-701 (1970).

20. R. Badugu, K. Nowaczyk, E. Descrovi, and J. R. Lakowicz, "Radiative decay engineering 6: Fluorescence on one-dimensional photonic crystals," Anal. Biochem. 442, 83-96 (2013).

21. H. Raether, Surface Plasmons (Springer-Verlag, 1988).

22. N. Danz, R. Waldhäusl, A. Bräuer, and R. Kowarschik, "Dipole lifetime in stratified media," J. Opt. Soc. Am. B 19, 412-419 (2002).

23. R. R. Chance, A. Prock, and R. Silbey, "Molecular fluorescence and energy transfer near interfaces," in Advance in Chemical Physics, I. Prigogine and S. A. Rice, eds. (John Wiley \& Sons, 1978), Vol. XXXVII, pp. 1-65.

24. C.-T. Tai, Dyadic Green's Functions in Electromagnetic Theory (Intext, 1971).

25. Y. Yuan and D. Axelrod, "Photobleaching with a Subnanosecond Laser Flash," J. Fluoresc. 4, 141-151 (1994).

26. M. W. Evans, J. Evans, W. T. Coffrey, and P. Grigolini, Molecular Dynamics and Theory of Broad Band Spectroscopy (Wiley, 1982).

27. R. Courant and D. Hilbert, Methods of Mathematical Physics (Interscience Publisher, Inc., 1953), Vol. 1. 
28. "https://www.thermofisher.com/," . 